Check for updates

Cite this: RSC Adv., 2020, 10, 512

Received 29th August 2019

Accepted 15th December 2019

DOI: $10.1039 / c 9 r a 06864 c$

rsc.li/rsc-advances

\section{Antimicrobial peptides from Bombyx mori: a splendid immune defense response in silkworms $\uparrow$}

\author{
Jannatun Nesa, ${ }^{a}$ Abdul Sadat, ${ }^{b}$ Danieli F. Buccini, ${ }^{c}$ Ahmet Kati, (D) de \\ Amit K. Mandal (D)*af and Octavio L. Franco (D)*cg
}

\begin{abstract}
Bombyx mori L., a primary producer of silk, is the main tool in the sericulture industry and provides the means of livelihood to a large number of people. Silk cocoon crop losses due to bacterial infection pose a major threat to the sericulture industry. Bombyx mori L., a silkworm of the mulberry type, has a sophisticated inherent innate immune mechanism to combat such invasive pathogens. Among all the components in this defense system, antimicrobial peptides (AMPs) are notable due to their specificity towards the invading pathogens without harming the normal host cells. Bombyx mori L. so far has had AMPs identified that belong to six different families, namely cecropin, defensin, moricin, gloverin, attacin and lebocin, which are produced by the Toll and immune deficiency (IMD) pathways. Their diverse modes of action depend on microbial pathogens and are still under investigation. This review examines the recent progress in understanding the immune defense mechanism of Bombyx mori based on AMPs.
\end{abstract}

\section{Introduction}

Sericulture is one of the prime agriculture-based industries in the world and it continues to flourish worldwide due to an everincreasing demand for raw silk. ${ }^{1}$ To fulfil this demand, silkworm rearing practice has undergone drastic changes over time in order to produce higher quality silk. ${ }^{2}$ At present, the Indian silk industry has a massive impact on global sericulture, holding the second position in the silk yield rankings. The Indian silk industry produced 21273 metric tons of raw mulberry silk in 2016-17 [3AR 2017]. But this production rate is insufficient to fulfil the national and international demand. In addition, the theoretical capacity in silk production per capita has not yet been reached..$^{1,3,4}$ To increase the production rate of high quality silk, cultivation of disease-free silkworms is of paramount importance.

${ }^{a}$ Chemical Biology Laboratory, Department of Sericulture, Raiganj University, Uttar Dinajpur, 733134, West Bengal, India. E-mail: amitmandalo8@gmail.com

${ }^{b}$ Insect Ecology and Conservation Biology Laboratory, Department of Sericulture, Raiganj University, Uttar Dinajpur, 733134, West Bengal, India

'S-INOVA Biotech, Post-Graduate Program in Biotechnology, Catholic University Dom Bosco, Campo Grande, Mato Grosso Do Sul, Brazil. E-mail: ocfranco@gmail.com

${ }^{d}$ Biotechnology Department, Institution of Health Science, University of Health Science, Istanbul, Turkey

${ }^{e}$ Medical Microbiology Department, Faculty of Medicine, Acibadem Mehmet Ali Aydinlar University, Istanbul, Turkey

${ }^{f}$ Centre for Nanotechnology Sciences, Raiganj University, Uttar Dinajpur, 733134, West Bengal, India

${ }^{g}$ Center of Proteomic and Biochemical Analysis, Post Graduate Program in Genomic Sciences and Biotechnology, Catholic University of Brasilia, Brasilia, Brazil

$\uparrow$ Dedicated to Prof. Ranadhir Chakraborty, University of North Bengal, on the occasion of his $57^{\text {th }}$ birthday.
The high mortality rate of silkworms due to disease is the main obstacle to high rates of silk production. ${ }^{2,5}$ The leaves that are fed are considered to be the main source of disease-causing infections and, thus, special emphasis is paid to the cleanliness of food and accommodation. ${ }^{2}$ Production of disease-resistant silkworm varieties has also drawn attention as an alternative approach. As silkworms lack an acquired immune system, they depend solely on their innate defense mechanism to protect themselves from pathogenic diseases. ${ }^{6}$ Antimicrobial peptides (AMPs) are one of the chief components of the innate immune system in B. mori. ${ }^{7}$ Upon pathogenic infection, AMPs are rapidly released into the haemolymph of the insect, and then they eliminate pathogens either by disrupting the cell membrane or by intracellular killing of the invading pathogen. ${ }^{8}$

The present review article aims to understand how various AMPs from the silkworm function against bacterial infections as a part of an immune defense strategy.

\section{Silkworm diseases}

Silkworms have been domesticated by the silk industry, and they cannot reproduce on their own. Indeed, reproduction demands human interference in the rearing room, which makes silkworms susceptible to various infectious diseases, such as pebrine, flacherie, grasserie and muscardine. ${ }^{2}$ All these infectious diseases are caused by microorganisms, including protozoa, bacteria, viruses and fungi (Table 1)..$^{3,4}$

\section{Gut microflora of Bombyx mori L.}

Bacterial microflora in the arthropod gut is closely related to the digestive capability of the host., ${ }^{9,10}$ The digestive tract of the 
Table 1 Microbial diseases of mulberry silkworm, B. Mori ${ }^{2-4}$

S1. no. Disease

Pathogen

BmNPV

Nuclear

polyhedrosis

Cytoplasmic

BmCPV

polyhedrosis

Infectious

flacherie

Densonucleosis

BmDNV1, BmDNV2, BmDNV3

2.

Bacteraemia
Bacterial
diseases
of digestive tract
Septicaemia

Toxicosis

3.

Mycosis
White

muscardine

Green

muscardine

Yellow

muscardine

Red muscardine

Orange

muscardine

Aspergillosis

4.

Protozoan

Pebrine

Staphylococcus sp.,
Sporosporellauvella,

Isariafumosoroseus

Sterigmatocystis japonica

Aspergillusflavus, Aspergillusoryzae

Streptococcus sp., Staphylococcus sp.,

Serratiamarcescens

Bacillus thringiensis var. Sotto

Metarhiziumanisopliae,

Isariafarinosa

Not reported

Not reported

Not reported

Prevalent

Nosemabombycis
Nosema $\mathrm{sp} . \mathrm{M}-11$
Nosema $\mathrm{sp} . \mathrm{M}-14$
Vairomorpha $\mathrm{sp} . \mathrm{M}-12$
Pleistomorpha $\mathrm{sp} . \mathrm{M}-24$
Pleistomorpha $\mathrm{sp} . \mathrm{M}-25$
Pleistomorpha $\mathrm{sp} . \mathrm{M}-27$
Thelohania $\mathrm{sp} . \mathrm{M}-32$
Leptomonassp

Summer and rainy season

Rainy season

Prevalent

Prevalent

All season

Prevalent

Prevalent

Not reported

Prevalent

Not reported

Not reported

Not reported

Not reported

Not reported
Summer and Prevalent rainy season green in colour arge disease specks aroun stigma and small on skin, mummified larvae yellow Develop red patches few hour before death; no external growth

Develop orange patches few hour before death; no external growth

Formation of light yellow coloured spores on surface with dirty brown

$\%$ loss of cocoons (2016-17)

20

Sluggish larvae with paler, translucent, wrinkled skin 
silkworm is quite simple, without any specialized structures, consisting of three regions: the foregut, midgut and hindgut. The midgut represents the majority of the digestive tract, while the foregut and hindgut constitute smaller portions. ${ }^{11}$ Silkworms are holometabolous insects and undergo five larval instars (referred to as L1 through L5), before metamorphosing to short-lived reproductive adults. Metamorphosis directly affects the intestinal microbiota. It is reported that the variety in bacterial species strikingly decreases in the adults. ${ }^{12}$ Kalpana et al. reported the presence of heterotopic bacterial population coupled with different larval stages of $B$. mori, and they related this finding with mulberry leaves fed to them. ${ }^{2}$ It was also revealed that there was more gut microflora in the middle region of the digestive tract of the late (fourth and fifth) instar larvae. The highest bacterial population was recorded in the digestive tract of fifth instar larvae $\left(13 \times 10^{6} \mathrm{CFU} \mathrm{g}^{-1}\right)$, whereas first instar larvae possess lower bacterial populations $\left(5.7 \times 10^{4}\right.$ $\mathrm{CFU} \mathrm{g}^{-1}$ ) in their gut. ${ }^{2}$

Silkworm gut microflora belongs to the belongs to the genera Bacillus, Corynebacterium, Micrococcus, Enterobacteriaceae, Moraxella, Alcaligenes, Acinetobacter and Aeromonas. Regarding the phenological and physiological properties, the gut microflora is dominated by rod-shaped bacteria rather than cocci, and by Gram positive bacteria rather than Gram negative. All bacterial populations are capable of glucose fermentation, and they mainly produce protease enzymes (in the form of caseinase and gelatinase), followed by amylase and lipase. ${ }^{10}$ Another study revealed that fifth instar larvae of B. mori possess both culturable facultative anaerobic bacteria and culturable obligatory anaerobic bacteria in the digestive tract suspension, which utilize polysaccharides as a carbon source. ${ }^{10,13}$ It was reported earlier that wild families of $B$. morisuch as Acronicta major
(Noctuidae) and Diaphania pyloalis (Pyralididae) share the same food niche. Due to the similar foraging behaviour in all these three species, they possess highly assorted but idiosyncratic microbiota. ${ }^{12}$ The mid gut microbial content of all these three taxa is dominated by Proteobacteria, Actinobacteria, Firmicutes and Bacteroidetes phyla (Fig. 1). ${ }^{12}$

Different bacteria coexist in the gut microflora through balanced symbiotic or antagonistic relationships. Antagonism is characterized by synthesis of the antimicrobial products of one microorganism against others that inhabit the same ecological niche. ${ }^{14}$

\section{Antimicrobial peptides in B. mori $\mathrm{L}$}

After the invasion of pathogens through the first line of defense (physical barrier) and their breakthrough into the insect's body, the second line of defense (innate immune system) comes into play. ${ }^{15}$ The innate immune system in the form of cellular and humoral response prevents spreading and multiplication of the invading pathogens inside the host. ${ }^{16}$ Cellular response includes hemocyte-mediated actions, such as encapsulation, nodulation and phagocytosis. ${ }^{17}$ Humoral response leads to production of melanin (melanization), generation of reactive oxygen species (ROS) and stimulation of AMPs production. ${ }^{7}$

AMPs are proteins with low molecular weight that exert a diverse array of defense mechanisms against fungi, bacteria and viruses. These biochemically active elements are the main components of the humoral defense system of various insects. They are the potential and imminent drugs of the future, mainly because their small size enables them to diffuse quickly through the plasma membrane of the target bacterial cells and activate the host's defense mechanism against microbes without developing resistance or toxic effects. ${ }^{18}$ Different

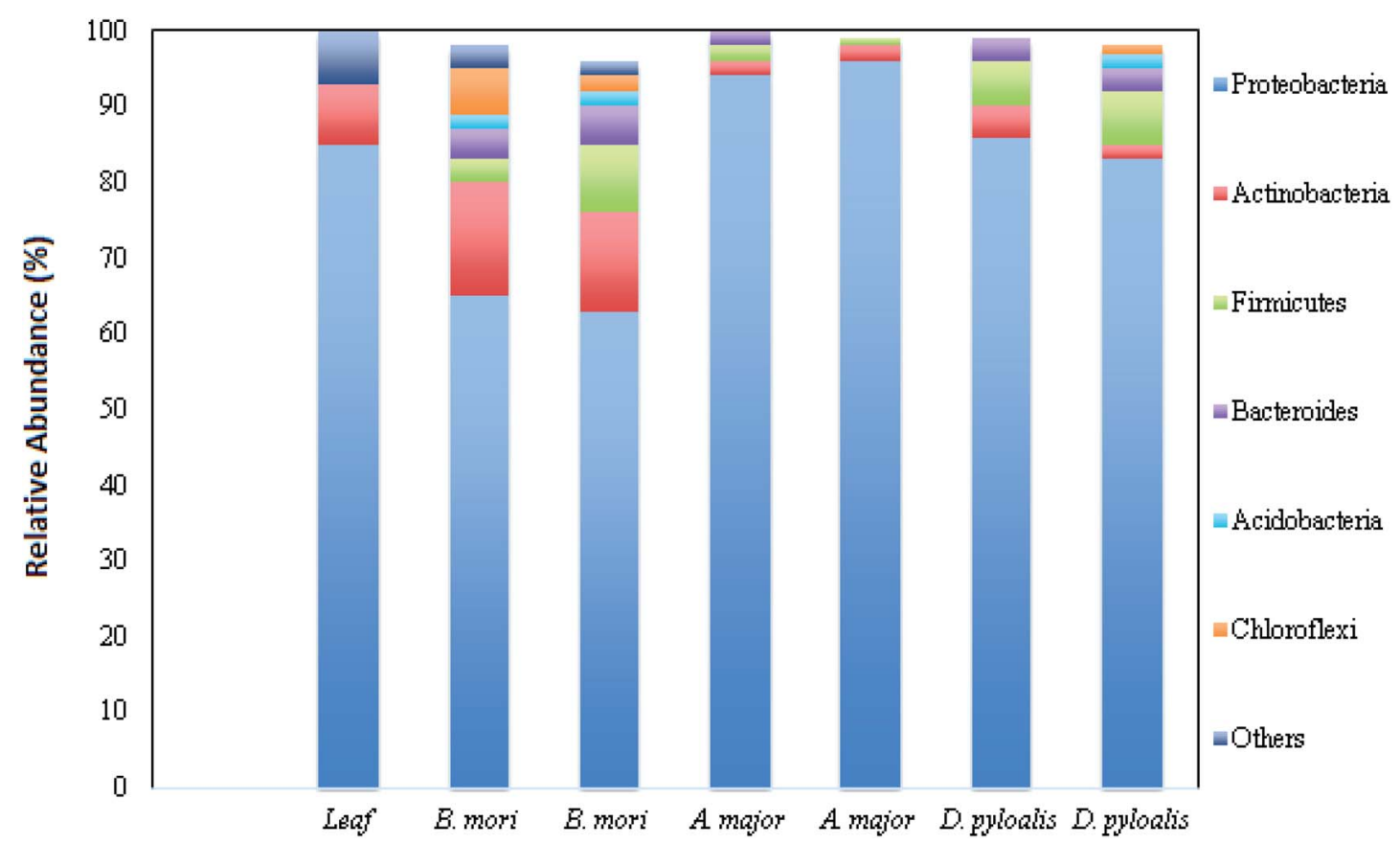

Fig. 1 Relative abundance of different bacterial phyla in host samples (this figure has been adapted from Chen et al., 2018 with permission from Springer Nature). ${ }^{12}$ 
experimental studies during the last few decades have established the presence of six families of AMP from B. mori viz.; cecropin, defensine, moricin, gloverin, lebocin and attacin (Table 2).

\section{Cecropin}

Cecropin was first isolated from the haemolymph of bacteriainfected giant silk worms (Hyalophoracecropia). ${ }^{19}$ Later, this AMP was found to be a part of the immune system in Antheraea pernyi and B. mori. Cecropins are cationic peptides that lack a cysteine residue with a strong basic $\mathrm{N}$-terminal linked to a neutral C-terminal by a flexible glycine-proline link. Insects possess three major types of cecropin; viz., cecropin A (37 amino acids), cecropin B (35 amino acids) and cecropinD (37 amino acids). In $B$. mori, 11 genes are responsible for the production of Bmcecropin, which occurred due to unequal cross-over events of the encoding gene. ${ }^{20}$ This AMP family is classified into five subtypes (Table 2), namely Bmcec A1 (2 genes), B6 (6 genes), C (1 gene), D (1 gene) and $\mathrm{E}$ (1 gene). The presence of six different clustered genes in chromosome 26 which encode amino acids for Bmcec B6 explains the phenomenon of gene duplication for that particular protein. ${ }^{21}$ Jiggins and Kim suggested that gene duplication increases AMP production to enhance immunity against microbial infections. ${ }^{22}$

Cecropins exhibit a broad range of antimicrobial properties against both Gram-positive and Gram-negative bacteria and fungi. ${ }^{18}$ Beside this, cecropins and their derivatives (SB-37 and Shiva) act as active suppressors of Trypanosoma and Plasmodium $^{23,24}$ and also inhibit the proliferation of tumour cell lines. ${ }^{25}$ Cecropin A causes the lysis of both Gram-positive and Gramnegative bacteria by the following mechanism: at first, Cecropin A binds to the negatively charged membrane lipids via its strongly positively charged N-terminus, and then it induces pore formation by its hydrophobic C-terminus, which then renders the membrane permeable, eventually leading to bacterial death. ${ }^{26}$ Among all the paralogs, BmcecB6 and BmcecD show the highest antimicrobial activity by inhibiting Bacillus bombysepticus, Bacillus subtilis, Bacillus thuringiensis, Bacillus thuringiensis galleriae, Escherichia coli, Serratia marcescens, Pseudomonas aeruginosa and Ralstoniasolaanacearum. ${ }^{\mathbf{2 0}}$ Besides, they are found to be active against the human leukaemia cell line. ${ }^{27}$ While these two paralogs remain inactive only against Staphylococcus aureus and Xanthomonas campestris. Bmcec $\mathrm{A} 1$ and BmcecE are active against five tested bacteria, and BmcecC has no inhibition activity against tested bacterial populations (Table 3 ). ${ }^{20}$

$\mathrm{Xia}$ et al. reported successful expression of the cecropin $\mathrm{XJ}$ gene in E. Coli and its inhibition of Gram-positive and Gramnegative bacteria, ${ }^{28,29}$ which opens up a new avenue for using cecropin against bacterial infections in silkworm and as a potent drug for broader therapeutic applications.

\section{Defensin}

An insect defensin was first isolated from the haemolymph of immunized flesh fly (Saecophoga peregrine). ${ }^{30}$ They are cationic peptides (Table 2) comprised of six conserved cysteine 


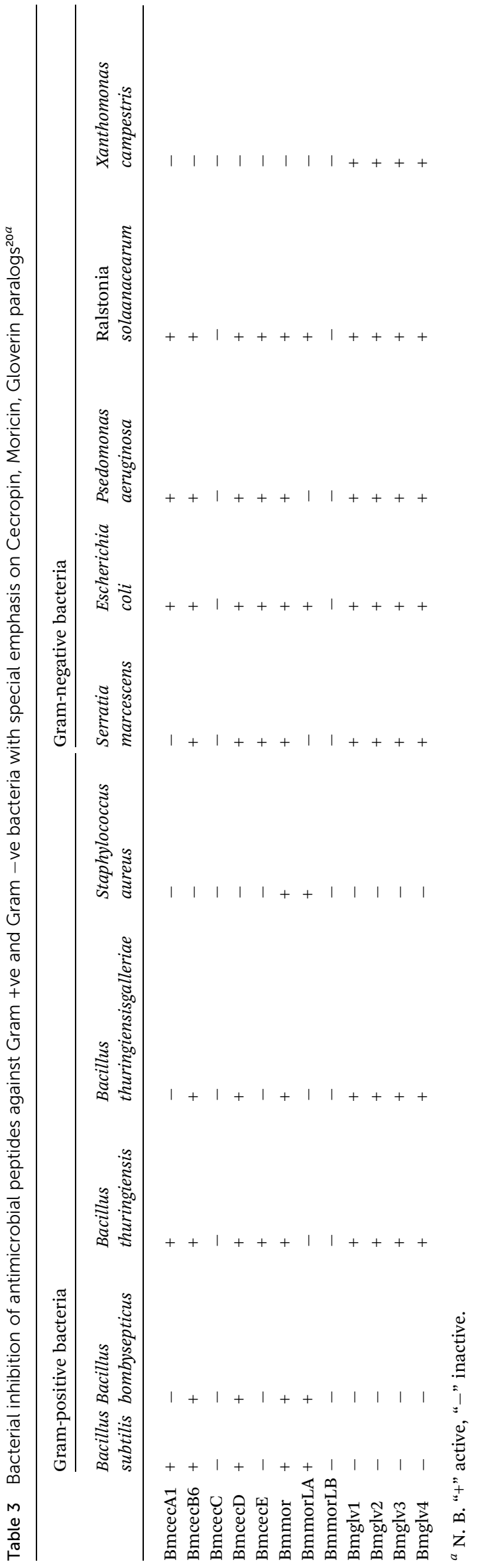

molecules which are responsible for the formation of three intra-molecular disulfide bonds. ${ }^{31}$ Phormicins, royalcins, sapecins and spodoptericis are different groups of the insect defensin family which were isolated and characterised from the haemolymph of the order Lepidoptera. ${ }^{32,33}$ These AMPs are mainly active against Gram-positive bacteria, including Micrococcus luteus, Aerococcus viridians, Bacillus megaterium, Bacillus subtilis, Bacillus thuringiensis, and Staphylococcus aureus. ${ }^{34}$

Wen et al. discovered the BmDefensinA gene from the B. mori genome, which is assumed to be related to insect defensins. ${ }^{35}$ Complete characterization of this gene revealed that the $5^{\prime}$ upstream region contains three conserved regulatory sequences; viz., NF-k $\beta$ binding, IL-6 responsive and GATA element. This BmDefensinA gene from B. mori translates into a large peptide which consists of an N-terminus signal peptide (22 amino acids), a pro-peptide (34 amino acids) and a mature peptide (36 amino acids) with molecular mass of $4 \mathrm{kDa}$. The mature peptide contains six conserved cysteine motifs, homologous to insect defensins. Unlike insect defensins, this mature BmDefA peptide is a novel anionic defensin with an isoelectric point of 4.2. This AMP is produced in large quantities in haemocytes, head, silk gland, fat body and ovary, and participates in both the defense mechanism and metamorphosis. ${ }^{18}$ Yoichi et al. isolated another peptide, namely BmDefB, homologous to and insect defensin with six conserved cysteine motifs, but only $27 \%$ amino acid similarities to the BmDefA peptide. ${ }^{36}$ The $B m D e f B$ peptide is largely synthesised in fat body and associated with protection against a broad spectrum of bacteria, including Escherichia coli and Bacillus subtilis. The Bm Defensin peptide forms large channels in the plasma membrane of bacterial cells and causes the efflux of cytosolic $\mathrm{K}^{+}$ions, partial depolarization of plasma membrane, decreased cytosolis of ATP and inhibition of respiration, eventually leading to bacterial cell death. ${ }^{37}$

\section{Moricin}

Moricin belongs to the AMP family that was first isolated from B. mori. Immunised B. mori produce this AMP in the haemolymph, and thus actively suppress the multiplication of Staphylococcus aureus. ${ }^{38}$ Moricin (encoded by multiple gene family) consists of a cationic peptide chain (42 residues long) formed by an amphipathic $\alpha$-helix with charged amino acids in the $\mathrm{N}$ terminal half, at an interval of every three to four amino acid residues. ${ }^{39,40}$ The B. mori genome harbours 12 moricin-coding genes that are synthesized into three subtypes (Table 2) viz., Bmmor (1 gene), moricin-like A (3 genes) and moricin-like B (8 genes). ${ }^{20}$ All the genes encoded mature moricin, which contains a positively charged C-terminus and an amphipathic alphahelical N-terminus without any post-translational modification. Lack of this post-translational modification made this moricin able to be synthesized chemically. ${ }^{18}$

This cationic peptide easily attaches to the negatively charged cell surface of bacteria, and the amphipathic $\alpha$-helical motif forms channels in bacterial plasma membrane that disrupt the ionic balance of that bacterial cell. ${ }^{41}$ Though it is active against both bacterial and fungal infection, its activity is recorded as higher against Gram-positive bacteria. The $\alpha$-helical 
motif of moricin plays a pivotal role in increasing the membrane permeability in order to kill bacterial pathogens. ${ }^{34}$ Lepidopteran-specific moricin shows a wide array of antibacterial activity, but this peptide from silkworm differs in its spectrum and activity. An in vitro experiment showed that moricin from B. mori (Bmmor), at dosages of 0.625-1.25 $\mu \mathrm{L} \mathrm{L}^{-1}$, exhibited antibacterial activities against $B$. bombysepticus, $B$. subtilis, B. thuringiensis, B. thuringiensis galleriae, E. coli, S. marcescens, $S$. aureus, $P$. aeruginosa and $R$. solaanacearum (Rd). Among the moricin family of B. mori, Bmmor exhibits the highest antibacterial toxicity $(p<0.01)$ followed by moricin-like A subtype. In contrast, moricin-like B subtype remains inactive against those tested bacteria. ${ }^{20}$

\section{Gloverin}

Gloverin was first isolated from pupal haemolymphs of the giant silk moth (Hyalaphora cecropia). Unlike other AMPs, gloverinis made up of glycine-rich (18.5\%) amino acids and devoid of cysteine. ${ }^{42}$ In addition, this AMP is only found in lepidopteran insects, including $B$. mori and Antheraea mylitta ${ }^{\mathbf{4 3 , 4 4}}$ Whole genome analysis of $B$. mori revealed that mulberry silkworm contains four genes (Table 2) which encode Bmglv 1, 2, 3 and $4 .^{20}$ These peptides are over-expressed in fat body of $B$. mori after induction with $E$. coli. ${ }^{45}$ The BmGlov gene contains an NF-kB like motif that provides a binding site in the upstream region of the gene. The Bmglv 1 gene is the ancestral one and evolved into Bmglv2-4 upon duplication in embryonic stage, indicating that the derived genes have gained embryonic expression and novel function. ${ }^{\mathbf{4 5 , 4 6}}$

Experimental work showed that gloverin actively inhibits mutant strains of E. coli (D21, Df21f2 and D22) that possess lipopolysaccharide (LPS) on their cell surface, and it was found to be inactive against smooth LPS containing E. coli strain. The product of the whole gene family significantly suppresses bacterial infection. ${ }^{\mathbf{1 8}}$ Bmglv family peptides showed an antibacterial effect on $B$. thuringiensis, B. thuringiensis galleriae, E. coli, $S$. marcescens, $P$. aeruginosa and $R$. Solaanacearum, except against $S$. aureus, B. bombysepticus and B. subtilis. ${ }^{20}$

\section{Attacin}

Attacin is a peptide chain (Table 2) with a molecular mass of 20$23 \mathrm{kDa}$ and was first isolated and characterised from the pupal haemolymph of bacterial immunized Hyalaphora cecropia. ${ }^{47}$ This AMP is expressed both in acidic and basic form with isoelectric points ranging from 5.7 to 8.3 , due to posttranslational modification of two parental pro-peptide sequences. Acidic attacin possesses a high content of aspartic acid, arginine and isoleucine, whereas basic attacin contains a high content of lysine, glutamic acid and tryptophan. ${ }^{18}$

Attacin shows antibacterial activity against E. coli, Stenotrophomonas maltophilia and Acinetobacter calcoaceticus. Unlike cecropin, attacin does not degrade the cell wall of the target bacteria, instead killing the bacteria by acting on growing cells and causing achain formation. Attacin restrains the synthesis of E. coli membrane proteins (OmpA, OmpC, OmpF and LamB), which alters the membrane structure and permeability. ${ }^{\mathbf{4 8 , 4 9}}$

\section{Lebocin}

Lebocin is a proline-rich peptide (Table 2) with $O$-glycosylated threonine (15-Thr) residue isolated from the haemolymph of immunized B. Mori. ${ }^{50}$ Glycosylation of the threonine residue of this AMP is crucial for its antibacterial activity. Four structurally related lebocin peptides were isolated and characterised, viz., lebocin 1, 2, 3 and 4, which are similar in peptide sequence (Table 2) but differ only in their sugar moiety. An in vitro study revealed that lebocin causes leakage of the lipid bilayer under low ionic conditions, suggesting active rupture of the bacterial membrane. ${ }^{50}$ But its exact mechanism is still under investigation, due to its low sensitivity and the need for a low ionic condition for its proper functioning. Lebocin 3 has a synergistic effect on cecropin $\mathrm{D}$, as their conjugation greatly increases the antibacterial activity of cecropin $\mathrm{D}$ in $B$. mori. ${ }^{51}$

\section{Molecular mechanism of AMP synthesis via Toll and IMD signalling pathway}

In silkworms, fungal or Gram-positive bacterial infection triggers activation of the Toll pathway (Fig. 2), resulting in the systemic production of AMPs. ${ }^{52,53}$ Cell wall components of invading fungi like $\beta$-glucan and, in Gram-positive bacteria in the form of Lysine-type peptidoglycan (Lys-PG), trigger the activation of serine protease (SP) cascades. ${ }^{54}$ The recognition of both $\beta$-glucan and/or Lys-PG may be mediated by Gram-negative binding protein (GNBP) 3 which is an extracellular recognition factor. ${ }^{55}$ The SP cascade triggers the activation of the Toll receptor ligand, Spatzle $(\mathrm{Spz})$ with the help of the Spatzleprocessing enzyme (SPE). ${ }^{56,57} \mathrm{Spz}$ (PRO Spz) is present in the cell membrane of the infected silkworm with hydrophobic Cterminal Spz region in inactive form. SPE induces proteolysis, which leads to conformational changes which expose the determinants that are critical for binding of the Toll receptor. ${ }^{58}$ After this conformational modification, the Spz-Toll complex binds to an adaptor protein, MyD88, through the intracellular TIR domain. ${ }^{59}$ Following this, a heterotrimeric complex (MyD88-Tube-Pelle) is formed, leading to phosphorylation and degradation of the IkB factor, cactus. ${ }^{60}$ In general, cactus is bound to the NF-kB transcription factor(s) like Dorsal and/or Dif, inhibiting its activity and nuclear localization. The activation and nuclear translocation of Dorsal and Dif requires degradation of cactus by the kinase activity of Pelle, ${ }^{61-63}$ leading to an increase in the synthesis of AMPs.

Peptidoglycan recognition proteins (PGRPs) were first identified and characterized in the silkworm Bombyx mori. ${ }^{64}$ These are $17 \mathrm{kDa}$ proteins with a strong affinity towards Peptidoglycans (PGs). The PGRPs are an evolutionarily conserved family of microbial recognition proteins/enzymes found in both insects and mammals. ${ }^{65}$ The PGRPs consist of a domain with homology to a PG-digesting enzyme known as $N$-acetylmuramyl-L-alanine amidase, which cleaves the bond between the lactyl group in $N$ acetylmuramic acid and L-alanine in the stem peptide of PG. PGRPs with amidase activity can degrade Gram-negative bacteria. The amidase activity is restricted to diaminopimelic acid-containing peptidoglycans (DAP-PG), which are most common inthe cell wall of Gram-negative bacteria. However, 


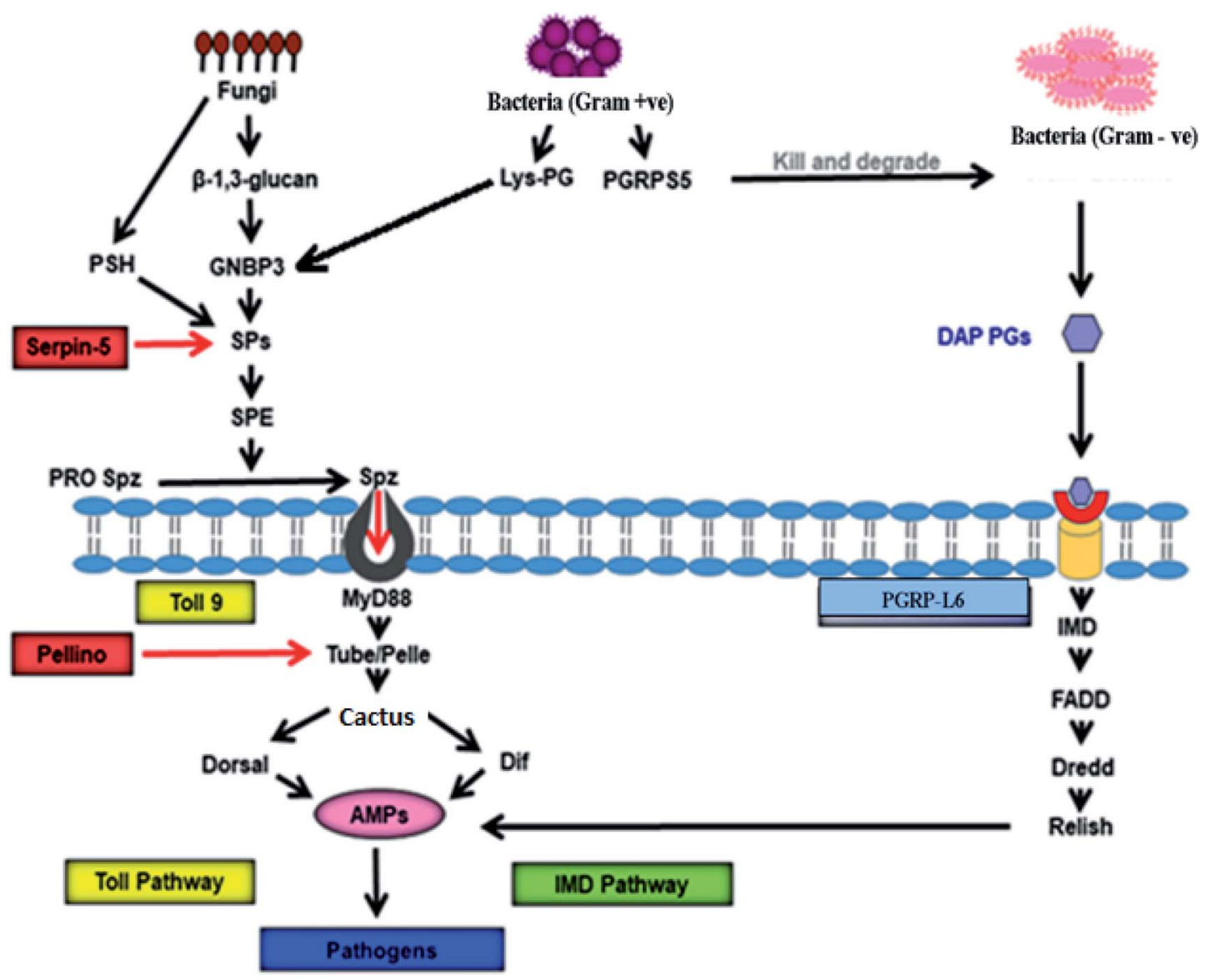

Fig. 2 Signalling pathway of antimicrobial activity achieved by AMPs in B. mori. GNBP-3 recognize $\beta$-1,3-glucan present on the cell wall of the invading fungi that activate Toll pathway through Toll9, using serine protease cascade, and persuade production of the AMPs that kill invading pathogens. On the other hand, invading Gram-positive bacteria produce Lys-PG, which also activates expression of AMPs via Toll9 using serine protease cascade. PGRPs recognise PGs of the Gram-positive and Gram-negative bacterial cell wall to induce AMP production through IMD pathway. PGRP-L6 in B. mori may act as intracellular receptors that control IMD pathway. GNBP: Gram-Negative Binding protein, PSH: persephone, SP: serine protease, SPE: Spatzle-processing enzyme, pro Spz: pro Spatzle, Spz: Spatzle, PG: peptidoglycan, Lys-PG: Lysis-PG, PGRP: peptidoglycan recognition protein, IMD: immune deficiency, DAP-PG: diaminopimelic acid-PGs, AMPs: antimicrobial peptides. ${ }^{52-65}$

PGRP binds to Lys-PG without any catalyzing activity in Grampositive bacteria. ${ }^{66}$ This catalyzing activity of PGRP probably prevents DAP-PG from activating the Toll pathway and initiates the expression of AMPs via the IMD pathway. ${ }^{65}$ Infection in Bombyx mori with Gram-negative bacteria simultaneously triggers the IMD pathway (Fig. 2). The intracellular IMD signalling pathway requires the formation of a receptor complex that includes IMD protein, FADD, and the caspase Dredd. ${ }^{67}$ With the help of caspase activity Dredd, it cleaves Relish (a drosophila NF-kB precursor protein) and causes its phosphorylation. After phosphorylation, the N-terminal NF-kB component of Relish translocates into the nucleus and triggers AMP gene expression several-fold to fight against Gram-positive bacteria. ${ }^{65}$

\section{Regulation of AMP production in silkworm}

AMP production is in direct step with the pathogenic infection, but many intrinsic factors regulate specific AMP production following immune challenges. Insects have rapid and transient activation of immune genes after microbial infection to produce effectors. When microorganisms reach the haemolymph, the recognized invaders occur for the first time due to cellular immune systems and humoral immune reactions. ${ }^{21}$ Modulation and signalling factors are stimulated, and signal transduction is caused only in specific tissues. ${ }^{21}$ The genes that encode effectors are activated by cascade signalling. ${ }^{8}$

It was reported earlier that different geographical types of silkworm (Japanese, Chinese, European and Tropical) are characterized by variable susceptibility to infectious pathogens. It was noted that European and Indian strains display the lowest sensitivity to E. mundtii and $S$. marcescens respectively. Although all four types of silkworm produce AMPs against both pathogens, European and Indian strains regulate their AMP production in a different manner for different pathogens. The European strain produces a specific composition of its AMP cocktail, with a more effective variant cecropin B6 isoform to suppress $E$. mundtii, while the Indian strain becomes resistant to $S$. marcescens with its prompt ability to activate the systemic transcription of AMPs. It is suggested that $B$. mori strains with a distinctive gene pool employ different strategies to fight 
bacterial infections, whose efficiency appears to be pathogendependent. ${ }^{68}$

Among AMPs, cecropin (Cec) includes five subtypes (A-E): the CecB subtype is active against Gram-positive and Gramnegative bacteria. $\mathrm{CecB}$ antimicrobial activity is related to interactions with bacterial membranes and pore formation. ${ }^{69}$ Further study revealed that $B$. mori cells, challenged against $P$. aeruginosa, expressed three classes of AMPs of which cecropin $\mathrm{B}$ isoform is of utmost important. ${ }^{70}$ The membranolytic activity for both $\mathrm{CecB}$ (Q53 CecB and $\mathrm{E} 53 \mathrm{CecB}$ ) isoforms on the $P$. aeruginosa outer membrane, followed by permeabilization of the inner membrane and subsequent disintegration of both, causes cytoplasmic content leakage. The Q53 CecB isoform differs from the E53 CecB variant in just one amino acid (glutamic acid replaced by glutamine) and showed the highest antimicrobial and membranolytic activity against $P$. Aeruginosa. ${ }^{70}$ The Q53 CecB isoform contains a critical factor in stabilizing the hydrophobic segment that interacts with the bacterial membrane, determining the highest antimicrobial activity of the whole peptide. Studies suggested, in fact, that the same AMP might possess different targets when tested against different pathogens. ${ }^{8}$

Recombinant DNA technology synthesized the gloverin2 peptide from the BmGlv2 gene in a prokaryotic system, which are more stable $\left(15-82.5{ }^{\circ} \mathrm{C}\right)$ and more active in preventing Gram-negative bacteria by disrupting cell integrity. ${ }^{69}$

\section{AMPs mediated defense mechanism in silkworm}

Silkworm produces ROS, phenoloxidase and AMPs as an array of defensive tools to combat bacterial infection. ${ }^{6,71}$ Among these three mechanisms, bacterial inhibition by AMPs is the most suitable because of its specificity towards invading pathogens. ${ }^{9}$ One property of insect innate immunity is the existence of an efficient systemic humoral immune response that fights microbial infections. This defense response consists of rapid and transient production of potent antibacterial and antifungal peptides, acting alone or in synergy when released into the haemolymph. These peptides are produced in the fat body (a functional homologue of the mammalian liver) and some blood cells. $^{72}$ The AMPs are then secreted into the haemolymph, where they are accumulated at high concentrations and further diffuse throughout the body. ${ }^{73}$ In insects, production of AMPs was first observed in Drosophila melanogastor. Expressions of AMPs (Fig. 2) are mainly under the control of nuclear factor-kB through Toll and IMD pathways, which was discussed earlier., ${ }^{721,65,74-77}$ Silkworm AMPs show broad spectrum antimicrobial activity in comparison to AMPs from D. melanogaster. ${ }^{28}$ Oral administration of heat-killed Pseudomonas aeruginosa cells and Serratia marcescens significantly activates production of AMPs against those strains. ${ }^{6}$ In contrast, oral administration heat-killed $S$. aureus remains unable to trigger production of AMPs against $P$. aeruginosa infection. This suggests that the presence of Gram-negative bacteria and fungi in the diet of silkworms activates the immune response against $P$. aeruginosa infection, but Gram-positive bacteria failed to trigger such responses. It was reported by in another study that $S$. marcescens and $P$. aeruginos $a$ are naturally occurring insect pathogens, in contrast to $S$. aureus. ${ }^{78-80}$ Thus, during co-evolution, insects may have developed a specific immune-activation strategy against such naturally occurring pathogenic bacteria.

In insects, AMP production through the IMD pathway needs the activation of NF-kB under regulation of the I kappa B kinase (IKK) complex. ${ }^{81}$ Oral administration of heat-killed $P$. aeruginosa cells into silkworm activates the synthesis of IKK $\gamma$ (a constituent of the IKK complex). Furthermore, these heat-killed orally administered bacteria, which acquire peptidoglycans, activate an insect cytokine known as paralytic peptide (PP), which leads to synthesis of AMPs through the IMD pathway. ${ }^{6,7,81,82}$

Defensin, at a dosage of $0.5 \mathrm{mg}$ per animal, actively inhibits pathogenic Staphylococcus aureus bacteria, which are found to be resistant towards methicillin. ${ }^{83}$ When wounded mice were treated with D2A21, an analogue to cecropin, 100\% survival was recorded when compared to that of control. ${ }^{84}$ Another group of synthetic cecropins (Shiva 11, d5c and Hecate) showed antibacterial activity against pathogens screened from infected contact lenses. Nowadays, these AMPs are used in a lens sterile solution to combat bacterial infections in conjunctiva. ${ }^{85}$ Silkworm AMPs show antimicrobial activity against a wide range of bacteria, such as Klebsiella pneumoniae, Klebsiella ozaenae, Shigella flexneri, Shigella sonnei, Staphylococcus aureus, Enterococcus faecalis, Staphylococcus epidermidis, Bacillus bombysepticus, Bacillus subtilis, Bacillus thuringiensis, Bacillus thuringiensis galleriae, Escherichia coli, Serratia marcescens, Pseudomonas aeruginosa, Ralstonia solaanacearum, Staphylococcus aureus and Xanthomons campestris., ${ }^{7,20,85}$ An earlier report showed that genetically engineered peptide Cecropin $\mathrm{XJ}$ is active against $S$. aureus with MIC of $0.4 \mu \mathrm{M}$, while other AMPs failed to exhibit this level of inhibition. ${ }^{28}$ Silkworm gut microflora plays a critical role in the synthesis of such AMPs. ${ }^{14}$ Silkworms actively suppress the infection against Yersinia pseudotuberculosis, B. bombyseptieus, E. coli, B. subtilis and $P$. aeruginosa with the help of AMPs that recognize bacterial peptidoglycans. The presence of gut microflora of $B$. mori induces expression of AMPs in fat body and haemocytes. ${ }^{6,77}$ The expression of attacin, cecropin, defensin, gloverin, leocin and moricin is accelerated by the oral administration of $P$. aeruginosa in gut microflora. ${ }^{6}$ It was also reported that specific proteins such as BmCPT1, BmPGRP-S5, and BmLBP collectively recognize $E$. coli in the midgut of $B$. mori to express different AMPs. ${ }^{86}$ On the other hand, B. bombyseptieus, a Gram-positive bacterium, activates the expression of a group of AMPs such as enbocin (which belongs to the cecropin family), lebocin, attacin, gloverin and moricin in the silkworm gut. ${ }^{87}$ Artificial feeding of B. mori with attenuated M. luteus leads to the increased production of cecropin D, cecropin E and gloverin 3 in body fat, while BmSerpin-5 reduces the production of these AMPs, down-regulating the Toll pathway by targeting BmHP6 and BmSP21. ${ }^{88}$ Laboratory experiments showed that recombinant BmSerpin-6, BmSerpin-15 down-regulated the expression of gloverin 2, cecropin $D$ and moricin in fat body and hemocytes of B. mori. ${ }^{88-90}$ In a separate study it was revealed that Bombyx mori nuclear polyhedrosis virus (BmNPV) infection in silkworm 
causes highest level of expression of attacin, whereas serpin-5 and cecropin-D exhibited a negative regulatory correlation. ${ }^{\mathbf{9 1}}$

The literature revealed that AMPs are a key component for immune defense against bacterial infection in silkworm. ${ }^{7,87,92-96}$ These observations stipulate that production of AMPs in silkworm through the Toll and IMD pathway is absolutely critical and remains unresolved. Hence, there is a need for detailed studies to understand such mechanisms, which will aid in providing more powerful tools against pathogenic multi-drugresistant microbes.

\section{Conclusion and future prospects ${ }^{97}$}

The present study looks at the molecular mechanism for the synthesis of AMPs in B. mori, which are the crucial effectors of the innate immune system, elucidating their development and their classification. These natural AMPs of B. mori are useful in illustrating the way in which the design of AMP variants for tackling the rising number of multi-drug-resistant infections, as a suitable substitute for conventional antibiotics.

\section{Author contributions}

J. N., A. S., D. F. B., A. K. wrote the manuscript with the help of A. K. M. and O. L. F.

\section{Conflicts of interest}

The authors declare no competing financial interest.

\section{Acknowledgements}

This work was supported by the Coordenação de Aperfeiçoamento de Pessoal de Nível Superior (CAPES), Conselho Nacional de Pesquisa e Desenvolvimento (CNPq), Fundação de Apoio à Pesquisa do Distrito Federal (FAPDF) and Fundação de Apoio ao Desenvolvimento do Ensino, Ciência e Tecnologia do Estado de Mato Grosso do Sul (FUNDECT).

\section{References}

1 S. R. Sarandavar, MS thesis, University of Agricultural Science, Dharwad, 2014.

2 S. Kalpana, A. A. M. Hatha and P. Laksmanaperumalsamy, Insect Sci. Its Appl., 1994, 15, 499-502.

3 Annual report, Central Silk Board, Ministry of Textile, Govt. of India, 2017.

4 Annual report, Central Silk Board, Ministry of Textile, Govt. of India, 2016.

5 B. B. Patnaik, D. H. Kim, H. O. Seung, Y. S. Song, N. D. M. Chanh, J. S. Kim, W. Jung, A. K. Saha, B. B. Bindroo and Y. S. Han, PLoS One, 2012, 7, e50900.

6 A. Miyashita, S. Takahashi, K. Ishii, K. Sekimizu and C. Kaito, PLoS One, 2015, 10, e0130486.

7 K. Chen and Z. Lu, Dev. Comp. Immunol., 2018, 83, 3-11. 8 J. A. Hoffmann, Nature, 2003, 426, 33-38.
9 J. M. Harris, L. J. Seiderer and M. I. Lucas, Microb. Ecol., 1991, 21, 277-296.

10 A. A. P. Anand, S. J. Vennison, S. G. Sankar, D. I. G. Prabhu, P. T. Vasan, T. Raghuraman, C. J. Geoffrey and S. E. Vendan, J. Insect Sci., 2010, 10, 1-20.

11 Anatomy of Silkworm Bombyx mori online, https:// hbmahesh.weebly.com/uploads/3/4/2/2/3422804/

4.anatomy.pdf, accessed June 2019.

12 B. Chen, K. Du, C. Sun, A. Vimalanathan, X. Liang, Y. Li, B. Wang, X. Lu, L. Li and Y. Shao, ISME J., 2018, 12, 22522262.

13 V. B. Khyade and R. M. Marathe, Global Journal of Bio-Science and BioTechnology, 2012, 2, 191-200.

14 E. Garcia-Gutierrez, M. J. Mayer, P. D. Cotter and A. Narbad, Gut Microbes, 2019, 10, 1-21.

15 D. Hultmark, Curr. Opin. Immunol., 2003, 15, 12-19.

16 B. Lemaitre and J. Hoffmann, Annu. Rev. Immunol., 2007, 25, 697-743.

17 C. A. Janeway Jr and R. Medzhitov, Annu. Rev. Immunol., 2002, 20, 197-216.

18 S. J. Islam, S. Ezbaruah and J. Kalita, J. Adv. Biol. Biotechnol., 2016, 9, 1-15.

19 D. Hultmark, A. Engström, H. Bennich, R. Kapur and H. G. Boman, Eur. J. Biochem., 1982, 127, 207-217.

20 W. Yang, T. Cheng, M. Ye, X. Deng, H. Yi, Y. Huang, X. Tan, D. Han, B. Wang, Z. Xiang, Y. Cao and Q. Xia, PLoS One, 2011, 6, e18109.

21 H. Tanaka, J. Ishibashi, K. Fujita, Y. Nakajima and A. Sagisaka, Insect Biochem. Mol. Biol., 2008, 38, 1087-1110.

22 F. M. Jiggins and K. W. Kim, Genetics, 2005, 171, 1847-1859.

23 S. C. Barr, D. Rose and J. M. Jaynes, J. Parasitol., 1995, 81, 974-978.

24 J. Boisbouvier, A. Prochnicka-Chalufour, A. R. Nieto, J. A. Torres, N. Nanard, M. H. Rodriguez, L. D. Possani and M. Delepierre, Eur. J. Biochem., 1998, 257, 263-273.

25 H. Suttmann, M. Retz, F. Paulsen, J. Harder, U. Zwergel, J. Kamradt, B. Wullich, G. Unteregger and M. Stockle, $B M C$ Urol., 2008, 8, 5-8.

26 S. M. Gregory, A. Cavenaugh, J. Velvet, P. Antje and F. F. A. Paulo, Biophys. J., 2008, 94, 1667-1680.

27 N. S. Parachin and O. L. Franco, Front. Microbiol., 2014, 5, 147.

28 L. Xia, F. Zhang, Z. Liu, J. Ma and J. Yang, Exp. Ther. Med., 2013, 5, 1745-1751.

29 B. J. Shahaji, O. A. Kumar, J. C. Balbhim, D. S. Mallikarjun and T. P. Ramrao, J. Adv. Bioinf. Appl. Res., 2015, 6, 8-22.

30 K. Matsuyama and S. Natori, J. Biol. Chem., 1988, 263, 1711217116.

31 Y. Xiao, A. L. Hughes, J. Ando, Y. Mastuda, J. F. Cheng, D. Skinner-Noble and G. A. Zhang, BMC Genomics, 2004, 5, 56.

32 S. Fujiwara, J. Imai, M. Fujiwara, T. Yaeshima, T. Kawashima and K. Kobayashi, J. Biol. Chem., 1990, 265, 11333-11337.

33 K. Yamada and S. Natori, Biochem. J., 1993, 291, 275-279.

34 H. Yi, M. Chowdhury, Y. D. Huang and X. Q. Yu, Appl. Microbiol. Biotechnol., 2014, 98, 5807-5822. 
35 H. Wen, X. Lan, T. Cheng, N. He, K. Shiomi, Z. Kajiura, Z. Zhou, Q. Xia, Z. Xiang and M. Nakagaki, Mol. Biol. Rep., 2009, 36(4), 711-716.

36 K. Yoichi, T. Hiromitsu, I. Jun, I. Takashi and Y. Inoru, Biosci., Biotechnol., Biochem., 2008, 72, 2353-2361.

37 L. Huang, T. Cheng, P. Xu, D. Cheng, T. Fang and Q. Xia, PLoS One, 2009, 12, e8098.

38 S. Hara and M. Yamakawa, J. Biol. Chem., 1995, 270, 2992329927.

39 T. Cheng, P. Zhao, C. Liu, P. Xu and Z. Gao, Genomics, 2006, 87, 356-365.

40 H. Tanaka, A. Sagikasa, K. Fujita, S. Furukuwa, J. Ishibashi and M. Yamakawa, Insect Biochem. Mol. Biol., 2012, 42, 474-481.

41 H. Hemmi, J. Ishibashi, S. Haraand and M. Yamakawa, FEBS Lett., 2002, 518, 33-38.

42 A. Axen, A. Carlsson, A. Engström and H. Bennich, Gloverin, Eur. J. Biochem., 1997, 247, 614-619.

43 S. Kawaoka, S. Katsuma, T. Daimon, R. Isono, N. Omuro, K. Mita and T. Shimada, Arch. Insect Biochem. Physiol., 2008, 67, 87-96.

44 A. S. Gandhe, K. P. Arunkumar, S. H. John and J. Nagaraju, BMC Genomics, 2006, 7, 184.

45 N. Mrinal and J. Nagaraju, J. Biol. Chem., 2008, 283, 2337623387.

46 H. Y. Yi, X. J. Deng, W. Y. Yanga, C. Z. Zhouc, Y. Caoa and X. Q. Yub, Insect Biochem. Mol. Biol., 2013, 43, 612-625.

47 M. Hedengren, K. Borge and D. Hultmark, Biochem. Biophys. Res. Commun., 2000, 279, 574-581.

48 A. Carlsson, P. Engstrom, E. T. Palva and H. Bennich, Infect. Immun., 991, 59, 3040-3045.

49 M. Sugiyama, H. Kuniyoshi, E. Kotani, K. Taniai, K. KadonoOkuda, Y. Kato, M. Yamamoto, M. Shimabukuro, S. Chowdhury, J. Xu, S. K. Choi, H. Katoaka, A. Suzuki and M. Yamakawa, Insect Biochem. Mol. Biol., 1995, 25, 385-392.

50 S. Hara and M. Yamakawa, Biochem. J., 1995, 310, 651-656.

51 M. Kangayam, K. M. Ponnuvel and M. Yamakawa, Curr. Sci., 2002, 83, 447-454.

52 C. Hetru and J. A. Hoffmann, Cold Spring Harbor Perspect. Biol., 2009, 1, a000232.

53 K. Aggarwal and N. Silverman, BMB Rep., 2008, 41, 267-277. 54 L. El Chamy, V. Leclerc, I. Caldelari and J. M. Reichhart, Nat. Immunol., 2008, 9, 1165-1170.

55 M. Gottar, V. Gobert, A. A. Matskevich, J. M. Reichhart, C. Wang, T. M. Butt, M. Belvin, J. A. Hoffmann and D. Ferrandon, Cell, 2006, 127, 1425-1437.

56 D. Morisato and K. V. Anderson, Cell, 1994, 76, 677-688.

57 D. S. Schneider, Y. Jin, D. Morisato and K. V. Anderson, Development, 1994, 120, 1243-1250.

58 C. J. Arnot, N. J. Gay and M. Gangloff, J. Biol. Chem., 2010, 285, 19502-19509.

59 T. Horng and R. Medzhitov, Proc. Natl. Acad. Sci. U. S. A., 2001, 98, 12654-12658.

60 H. Sun, B. N. Bristow, G. Qu and S. A. Wasserman, Proc. Natl. Acad. Sci. U. S. A., 2002, 99, 12871-12876.

61 L. P. Wu and K. V. Anderson, Nature, 1998, 392, 93-97.
62 P. Towb, A. Bergmann and S. A. Wasserman, Development, 2001, 128, 4729-4736.

63 H. R. Huang, Z. J. Chen, S. Kunes, G. D. Chang and T. Maniatis, Proc. Natl. Acad. Sci. U. S. A., 2010, 107, 83228327.

64 M. Ochiai and M. Ashida, J. Biol. Chem., 1999, 274, 1185411858.

65 T. Kaneko and N. Silverman, Cell. Microbiol., 2005, 7, 461469.

66 C. I. Chang, S. S. Pili-Floury, M. Herve, C. Parquet, Y. Chelliah and B. Lemaitre, PLoS Biol., 2004, 2, E277.

67 S. Hu and X. Yang, J. Biol. Chem., 2000, 275, 30761-30764.

68 O. Romoli, A. Saviane, A. Bozzato, P. D'Antona, G. Tettamanti, A. Squartini, S. Cappellozza and F. Sandrelli, Sci. Rep., 2017, 7(1), 1048.

69 Q. Wang, P. Guo, Z. Wang, H. Liu, Y. Zhang, S. Jiang, W. Han, Q. Xia and P. Zhao, Int. J. Mol. Sci., 2018, 19(8), E2275.

70 O. Romoli, S. Mukherjee, S. A. Mohid, A. Dutta A, A. Montali, E. Franzolin, D. Brady, F. Zito, E. Bergantino, C. Rampazzo, G. Tettamanti, A. Bhunia and F. Sandrelli, ACS Infect. Dis., 2019, 5(7), 1200-1213.

71 Y. Yasuhara, Y. Koizumi, C. Katagiri and M. Ashida, Arch. Biochem. Biophys., 1995, 320, 14-23.

72 D. Ferrandon, Nat. Rev. Immunol., 2007, 7, 862-874.

73 R. A. B. Ezekowitz and J. A. Hoffmann, Innate immunity, Humana Press, Totowa: NJ, 2002.

74 S. Rutschmann, A. Kilinc and D. Ferrandon, J. Immunol., 2002, 168, 1542-1546.

75 M. Yamakawa and H. Tanaka, Dev. Comp. Immunol., 1999, 23, 281-289.

76 Y. Kaneko, S. Furukawa, H. Tanaka and M. Yamakawa, Biosci., Biotechnol., Biochem., 2007, 71, 2233-2241.

77 Y. Kaneko, H. Tanaka, J. Ishibashi, T. Iwasaki and M. Yamakawa, Biosci., Biotechnol., Biochem., 2008, 72, 2353-2361.

78 P. A. Grimont and F. Grimont, Annu. Rev. Microbiol., 1978, 32, 221-248.

79 N. Vodovar, M. Vinals, P. Liehl, A. Basset, J. Degrouard and P. Spellman, Proc. Natl. Acad. Sci. U. S. A., 2005, 102, 11414-11419.

80 S. M. Braxton, D. W. Onstad, D. E. Dockter, R. Giordano, R. Larsson and R. A. Humber, J. Invertebr. Pathol., 2003, 83, 185-195.

81 B. Lemaitre, Nat. Rev. Immunol., 2004, 4, 521-527.

82 K. Ishii, H. Hamamoto, M. Kamimura, Y. Nakamura, H. Noda and K. Imamura, J. Biol. Chem., 2010, 285, 2863528642.

83 M. Yamada, K. Nakamura, H. Saido-Sakanaka, A. Asaoka, M. Yamakawa, Y. Yamamoto, Y. Koyama, K. Hikosaka, A. Shimizu and Y. Hirota, J. Vet. Med. Sci., 2005, 67, 10051011.

84 W. S. Jang, H. N. Kim, Y. S. Lee, M. H. Nam and I. H. Lee, FEBS Lett., 2002, 521, 81-86.

85 L. B. Sousa, M. J. Mannis, I. R. Schwab, J. Cullor, H. Houstani, W. Smith and J. Jaynes, The use of synthetic cecropin (D5C) in disinfecting contact lens solutions, CLAO J., 1999, 22, 114-117. 
86 J. Liang, T. Wang, Z. Xiang and N. He, Insect Biochem. Mol. Biol., 2015, 58, 76-88.

87 L. Huang, T. Cheng, P. Xu, D. Cheng, T. Fang and Q. Xia, PLoS One, 2009, 4, e8098.

88 J. L. Li, L. Ma, Z. Lin, Z. Zou and Z. Q. Lu, Insect Biochem. Mol. Biol., 2016, 73, 27-37.

89 Y. Li, P. Zhao, H. Liu, X. Guo, H. He, R. Zhu, Z. Xiang and Q. Xia, Insect Biochem. Mol. Biol., 2015, 57, 11-19.

90 B. Li, H. Z. Yu, C. J. Ye, Y. Ma, X. Li, T. Fan, F. S. Chen and J. P. Xu, Gene, 2017, 610, 64-70.

91 W. T. Dong, X. D. Ling, L. F. Xiao, J. J. Hu, X. X. Zhao, J. X. Liu and Y. Zhang, Microb. Pathog., 2019, 130, 137-145.
92 K. K. Chen, L. Zhou, F. Chen, Y. Peng and Z. Q. Lu, Dev. Comp. Immunol., 2016, 61, 126-135.

93 S. Wu, X. Zhang, Y. He, J. Shuai, X. Chen and E. Ling, Dev. Comp. Immunol., 2010, 34, 1191-1198.

94 C. Y. Zhou, X. F. Zha, C. Liu, M. J. Han, L. Y. Zhang, P. P. Shi, H. Wang, R. W. Zheng and Q. Y. Xia, Insect Sci., 2016, 23, 502-512.

95 L. Zhi, Ma. Yan, L. Xuan, L. Yi Li and F. Dai, Anim. Biol., 2019, 69(4), 391-410.

96 S. Panthee, A. Paudel, H. Hamamoto and K. Sekimizu, Front. Microbiol., 2017, 8, 373.

97 O. Fleitas and O. L. Franco, Front. Microbiol., 2016, 7, 381. 